\title{
Monte Carlo study of hard pentagons
}

\author{
Tanja Schilling,* Sander Pronk, ${ }^{\dagger}$ Bela Mulder, and Daan Frenkel \\ FOM Institute for Atomic and Molecular Physics, Kruislaan 407, 1098 SJ Amsterdam, The Netherlands
}

(Received 1 November 2004; published 25 March 2005)

\begin{abstract}
How does a liquid freeze if the geometry of its particles conflicts with the symmetry of the crystal it should naturally form? We study this question in the simplest model system of particles exhibiting such a symmetry mismatch: hard pentagons in two dimensions. Using isobaric and isotensic Monte Carlo simulations we have studied the phase behavior of hard pentagons. On increasing the pressure from the homogeneous and isotropic low-density phase, the system first exhibits a rotator phase (plastic solid) with a triangular lattice structure. At higher densities it undergoes a weak first-order phase transition into a "striped" phase composed of alternating rows of oppositely pointing particles. This phase is analogous to the "striped" phase in the compressible antiferromagnetic Ising model on a triangular lattice and is an example of systems in which frustration due to the mismatch in symmetries is released by an elastic coupling to the lattice. In order to pursue this analogy we also consider hard heptagons, showing that in this case the decrease in symmetry mismatch indeed leads to a shift in the transition densities to higher values and a weakening of the transition.
\end{abstract}

DOI: 10.1103/PhysRevE.71.036138

PACS number(s): 64.60.-i, 61.20.Gy, 64.70.Dv, 05.10.Ln

\section{BACKGROUND}

Shape is one of the main characteristics of molecules. Indeed, simple models in which molecules are represented by hard objects of a given shape without any interaction apart from their excluded volume have been very successful in the analysis of the phase behavior of liquids, liquid crystals [1,2], and the solid phases that develop from them. In most cases heuristic packing arguments can be used as a safe guide to predict possible (liquid-)crystalline structures in these systems.

What happens, however, if the particle geometry does not naturally "fit" into one of the crystal structures into which the liquid tends to freeze? The simplest objects which seem to fulfill this requirement are two-dimensional (2D) particles with fivefold symmetry. At low densities they act effectively like hard disks, which would form hexagonal structures. However, at high densities one expects the fivefold symmetry to strongly interfere with hexagonal ordering. In such a system the ultimate stable crystalline phase is thus the result of a subtle tug-of-war between the spatial and orientational degrees of freedom. A system of this type was first considered by Brańka and Wojciechowski who studied a system of hard pentamers (objects made from five circular disks arranged with their centers on the vertices of a pentagon) in both mechanical simulations [3] and computer simulations [4-6]. They indeed found that on compression their system undergoes two successive transitions: first to an orientationally disordered plastic solid phase with hexagonal symmetry and finally to a dense phase with rectangular unit cell in which the pentamers are orientationally ordered with respect to the lattice vectors. Due to computational limitations, these

\footnotetext{
*Current address: Institut für Physik, Johannes GutenbergUniversität D-55099 Mainz, Germany.

${ }^{\dagger}$ Current address: Physical Biosciences Division, Lawrence Berkeley National Laboratory, Berkeley, CA 94720, USA.
}

authors studied relatively small systems composed of $N$ $=120$ particles. Wojciechowski and co-workers later considered larger systems of up to $N=896$ but then focussed on the elastic properties of the solid phases exclusively [7].

Here we reexamine these questions using what is arguably the simplest shape in the class of "misfit" particles-i.e., hard pentagons. Our main motivation is the fact that phase transitions in 2D are notoriously sensitive to system size. Current computational capabilities allow us to study a significantly larger system size-specifically, $N=4763$ or 30 times that of Refs. [4,5]—using the isotensic ensemble, thus ruling out that the phases observed are artifacts of a specific simulation box geometry. Moreover, we have determined both orientational and positional order parameters and their coupling in the dense phases, thus allowing a much more detailed analysis of the transitions concerned. Finally, we interpret the observed high-density phase in the context of earlier work on "soft" frustrated systems, in which elastic deformation of an underlying crystal lattice serves to relax not fully satisfiable energetic or entropic constraints. The paradigmatic example is the Ising antiferromagnet on a deformable triangular lattice [8] whose low-temperature phase is readily mappable onto the high-density phase of hard pentagons. In the lattice systems one can independently vary the direct interaction between the spins and coupling to the lattice through an elastic modulus, allowing predictions of the nature of the phase transition. In hard-particle systems a change in particle shape will lead to intertwined changes in both the orientational and positional couplings. In order to assess this effect we also briefly study hard heptagons.

The outline of the paper is as follows. In Sec. II A we first define the order parameters by which we have identified the structures obtained in the simulations. In Sec. II B the measured data are presented and in Sec. II C we compare the freezing behavior to the transition in the compressible antiferromagnetic Ising model on a triangular lattice. In Sec. II D we explore this connection further by briefly considering the behavior of hard heptagons. Section III sums up our conclusions. 


\section{RESULTS}

We have performed both isobaric and isotensic Monte Carlo (MC) simulations of hard pentagons. In an isotensic simulation the pressure tensor is imposed instead of the pressure. The system is allowed to relax to the pressure tensor by sampling the box shape. Because the box shape is not necessarily rectangular, the system may form crystalline phases which would not have been commensurate with a rectangular box [9-11]. The simulations were performed with 4736 pentagons. Equilibration took $1.5 \times 10^{6} \mathrm{MC}$ steps per particle (sweeps) and production runs were of $1.0 \times 10^{6}$ sweeps. Results are given in length units of $\sigma$ (diameter of pentagon perimeter) and energy units of $k_{B} T$.

\section{A. Definition of the order parameters}

We have measured several quantities which reflect the positional or orientational ordering of the system. Some of these are the usual order parameters for studying 2D melting [12].

We define the local values of the order parameters at location $\mathbf{x}_{j}$, which is the location of pentagon $j$. The global $\operatorname{order} A$ for the local order parameter $a\left(\mathbf{x}_{j}\right)$ is then defined as

$$
A=\frac{1}{N}\left|\sum_{j=1}^{N} a\left(\mathbf{x}_{j}\right)\right|
$$

(we use absolute values because most order parameters have imaginary components).

First, the particle orientational order parameter $\phi_{n}\left(\mathbf{x}_{i}\right)$ is defined as

$$
\phi_{n}\left(\mathbf{x}_{j}\right)=e^{i n \theta_{j}},
$$

where $\theta_{j}$ is the angle between an arbitrary axis from the center to one of the vertices of pentagon $j$ and an arbitrary fixed axis. As a convenient measure of absolute orientation, which removes the fivefold degeneracy inherent in this definition of the orientation, we can use $\bar{\theta}_{j}=\theta_{j} \bmod (2 \pi / 5)$.

For bond-orientational order we use

$$
\psi_{n}\left(\mathbf{x}_{j}\right)=\frac{1}{N_{j}} \sum_{k=1}^{N_{j}} e^{n i \theta_{j k}},
$$

where $N_{j}$ is the number of neighbors of particle $j$, the sum is over the neighbors $k$ of $j$, and $\theta_{j k}$ is the angle between an arbitrary fixed axis and the line connecting particles $j$ and $k$. Two particles are neighbors if they share an edge in the Voronoi tesselation of the set of points that are the centers of the pentagons.

In a crystal, the (complex) sum

$$
\Psi_{n} e^{i \omega}=\frac{1}{N} \sum_{j=1}^{N} \psi_{n}\left(\mathbf{x}_{j}\right)
$$

for the appropriate value of $n$ determines an overall orientation $\omega$ of the crystal. This orientation can then be used with the lattice spacing - inferred from the density - to construct a reciprocal lattice vector $\mathbf{G}$ of the appropriate crystal which then allows the calculation of the positional order:

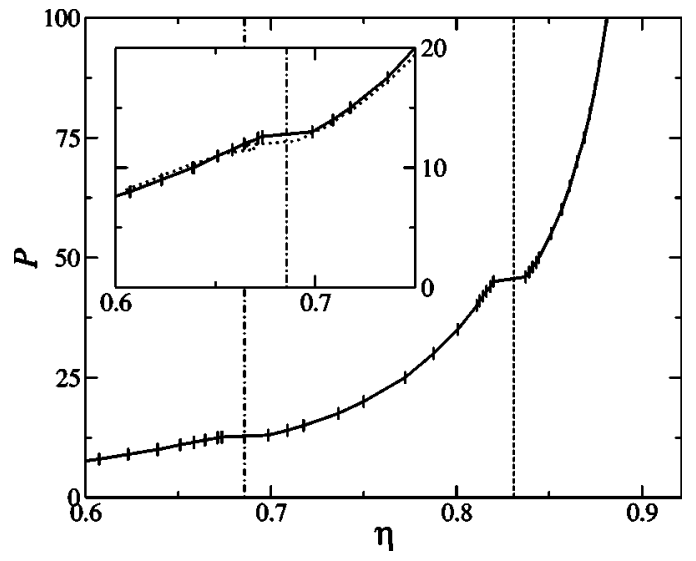

FIG. 1. Pressure $P$ versus packing fraction $\eta$ for hard pentagons: The equation of state shows two discontinuities: one at a low density (see the inset) and one at a higher density. The dotted line is a 2D hard-disk equation of state with both pressure and density scaled by a constant factor so as to optimally match the pentagon results.

$$
S(\mathbf{q}=\mathbf{G})=\frac{1}{N} \sum_{k=1}^{N} e^{i \mathbf{G} \cdot \mathbf{x}_{k}}
$$

To get an indication of the coupling between the neighbor position and particle orientation, the following bond-particle orientational parameter was used:

$$
\begin{aligned}
\Psi_{m} \Phi_{n}^{*} & \equiv \frac{1}{N}\left|\sum_{j=1}^{N} \psi_{m}\left(\mathbf{x}_{j}\right) \phi_{n}^{*}\left(\mathbf{x}_{j}\right)\right| \\
& =\frac{1}{N}\left|\sum_{j=1}^{N}\left\{\frac{1}{N_{j}} \sum_{k=1}^{N_{j}} e^{i m \theta_{j k}}\right\} e^{-n \theta_{j}}\right|,
\end{aligned}
$$

where, again, the inner sum is over the neighbors $k$ of $j$.

\section{B. Phase behavior}

In Fig. 1 the pressure $P$ is plotted versus the packing fraction $\eta$. For $2 \mathrm{D}$ particles $\eta$ is defined as the fraction of the total area covered and provides a convenient particle-shapeindependent dimensionless measure of the density. There are two discontinuities in packing fraction which could indicate first-order phase transitions. The high-density phase transition shows a clear density gap, while the nature of the lowdensity transition is not as clear. There the situation is similar to $2 \mathrm{D}$ hard-disk melting, where, due to the finite-size effects on the simulation, it is difficult to decide between a firstorder phase transition and a Kosterlitz-Thouless-HulperinNelson-Young (KTHNY) dislocation-unbinding melting scenario involving a hexatic phase (see, for example, [12]). The similarity between the present case and the hard-disk fluidsolid phase transition can be seen in the inset of Fig. 1, where the scaled hard-disk equation of state (dotted line) is superimposed on the pentagon equation of state (solid line).

The density dependence of the order parameters at the low-density transition is shown in Fig. 2. The sixfold bond order $\Psi_{6}$ and the structure factor of the corresponding lattice $S\left(\mathbf{q}_{\text {hex }}\right)$, where $\mathbf{q}_{\text {hex }}$ is the reciprocal lattice vector of the tri- 


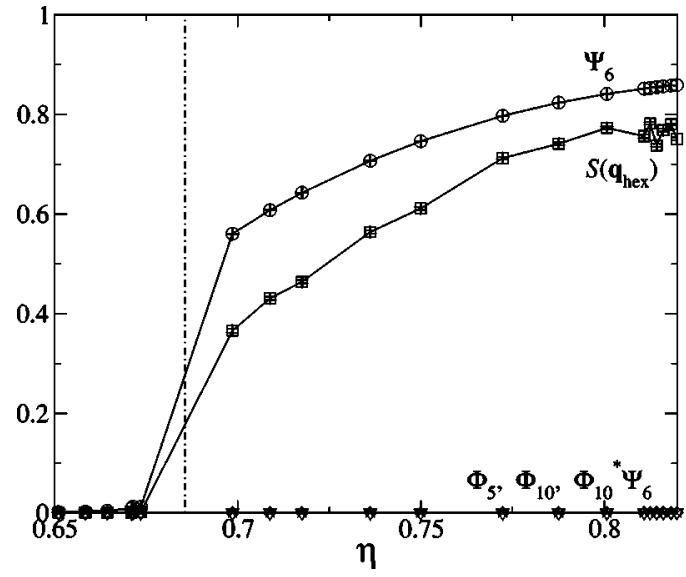

FIG. 2. Order parameters at the low-density phase transition for hard pentagons: The sixfold bond order $\Psi_{6}$ and the structure factor of the corresponding triangular lattice $S\left(\mathbf{q}_{\text {hex }}\right)$ increase.

angular lattice, increase. The particle positions are frozen onto a triangular lattice. The orientational parameters $\Phi_{n}$, however, remain close to zero; there is no orientational ordering at this transition. Also the cross coupling between the sixfold-symmetric crystal field and the fivefold-orientational symmetry of the particles, as measured by the order parameter $\Phi_{30}$, remains negligible (see Fig. 3). Of course, there are definite position-orientation correlations in this rotator phase, which have been studied in detail in Ref. [5] for the pentamers, but these do not build up to any appreciable net orientational order.

At the high-density transition (see Fig. 3) the bond order $\Psi_{6}$ increases further, but the corresponding structure factor $S\left(\mathbf{q}_{\text {hex }}\right)$ drops to zero. The system still has sixfold bond order, but the particles are no longer positioned in a regular triangular lattice. At the same transition orientational ordering sets in. In particular, $\Phi_{10}$ grows strongly. However, $\Phi_{5}$ remains effectively equal to zero. The reason why we observe only 10 -fold (in general, $10 n$-fold) ordering is that the particles align in "antiparallel" fashion, canceling $\Phi_{5}$ exactly. Keeping trend with the increased crystallinity, as signaled by the increasing solid structure factor $S\left(q_{c p}\right)$, the order parameter $\Phi_{30}$ now also develops finite values.

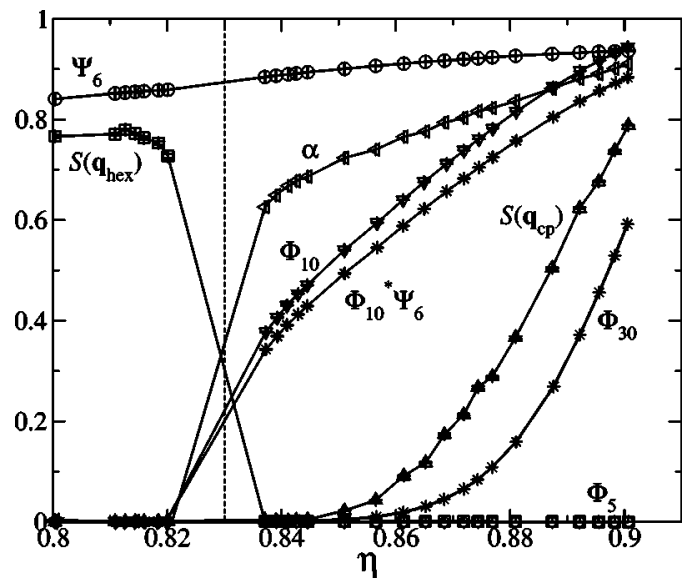

FIG. 3. Order parameters at the (higher-density) rotator-crystal phase transition for hard pentagons. For definitions see the text.
Together with the absolute orientational order, the coupling between orientational and bond-orientational order, in this case $\Phi_{10}^{*} \Psi_{6}$ goes from zero to a finite value at the transition. The structure that forms is a striped phase of antiparallelly packed pentagons, as the snapshot in Fig. 4 shows.

To our knowledge, there exists no proof that the regular arrangement of Fig. 4 (shown schematically in Fig. 5) is the densest possible pentagon packing on a plane, but it has been conjectured by Henley [13]. Our simulations and the experiments by Duparcmeur et al. [14] spontaneously form this arrangement at the highest pressures. The arrangement itself, as shown in Fig. 5, is crystallographically speaking a rectangular phase with a unit cell of size

$$
\begin{gathered}
a_{x}^{\mathrm{cp}}=\sigma \frac{5+\sqrt{5}}{8} \approx 0.905 a_{x}^{\mathrm{hex}}, \\
a_{y}^{\mathrm{cp}}=\sigma \sqrt{\frac{9}{32}(5+\sqrt{5})} \approx 0.824 a_{x}^{\text {hex }},
\end{gathered}
$$

where $a_{x}^{\text {hex }}$ and $a_{y}^{\text {hex }}$ are the equivalent hexagonal unit-cell dimensions. The two particles are located at

$$
\begin{gathered}
\mathbf{r}_{0}=(0,0), \\
\mathbf{r}_{1}=\left(\sigma\left[\frac{1}{16}+\frac{3}{16} \sqrt{5}\right], \frac{a_{y}^{\mathrm{cp}}}{2}\right),
\end{gathered}
$$

with a maximum packing fraction of 0.92131 . For reasons that will become clear in the following we prefer, however, to look upon this phase as a distorted hexagonal packing.

Figure 3 also shows the density dependence of the appropriate structure factor $S\left(\mathbf{q}_{\mathrm{cp}}\right)$ for this type of distorted hexagonal lattice. The figure clearly shows that this order parameter starts to grow at the phase transition. In addition we also followed the behavior of the "aspect-ratio order parameter," defined as

$$
\alpha=1-\left(\frac{a_{y}}{a_{x}}-\frac{a_{y}^{\mathrm{cp}}}{a_{x}^{\mathrm{cp}}}\right) /\left(\frac{a_{y}^{\mathrm{hex}}}{a_{x}^{\mathrm{hex}}}-\frac{a_{y}^{\mathrm{cp}}}{a_{x}^{\mathrm{cp}}}\right),
$$

where $a_{x}$ and $a_{y}$ are the measured lengths of the unit-cell edges corresponding to $a_{x}^{\mathrm{cp}}$ and $a_{y}^{\mathrm{cp}}$ in Fig. 5. This order parameter goes from 0 to 1 as the lattice distorts from hexagonal to close-packed structure, as can be seen in Fig. 3.

Upon compression from the rotator phase, the system forms a close-packed crystal with a large concentration of defects in the form of kinks in the stripes of the parallelly aligned pentagons, similar to what the pentagon packings of Duparcmeur et al. [14] showed. In addition, hysteresis prevents crystallization until pressures are well beyond those at which the crystal melts (in contrast to Ref. [4], where the system crystallized easily because of finite-size effects).

The size of the density gap, the hysteresis, and the jumps in the order parameter values (especially the one in $\alpha$ ) strongly suggest a first-order phase transition between the rotator phase and the close-packed crystalline phase. Given the large number of particles involved it is unlikely that it is a finite-size effect. 

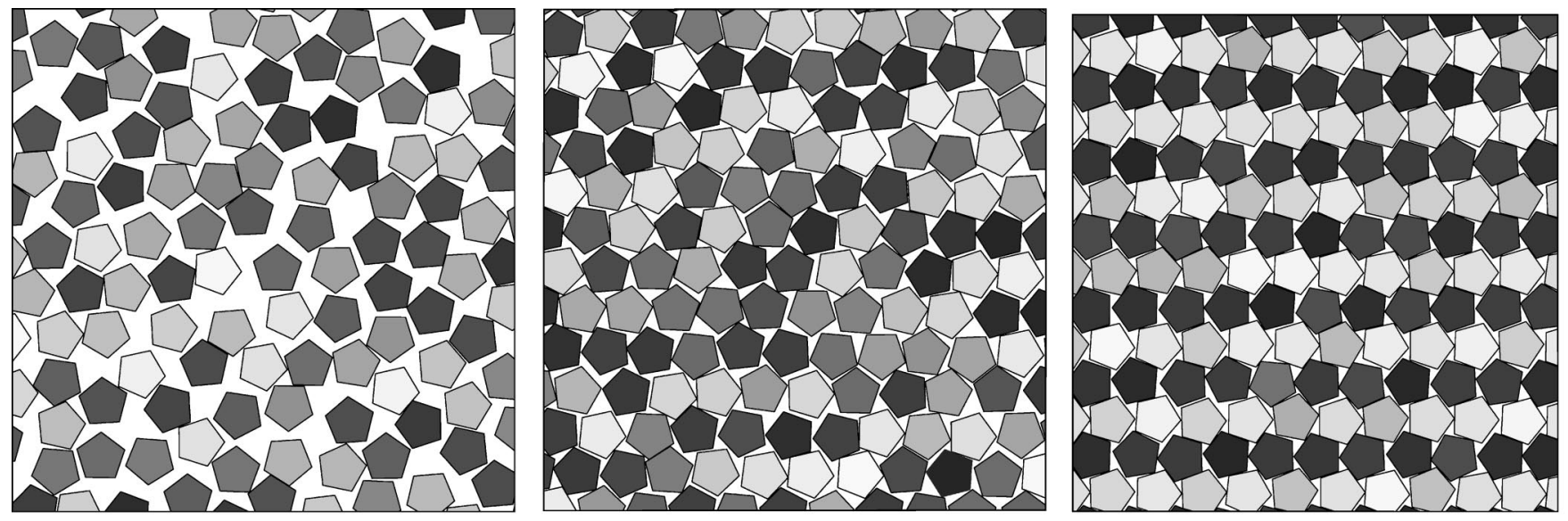

FIG. 4. Representative snapshots of the three phases. Fluid phase (left), rotator crystal (middle), and high-density crystal (right). The pentagons are shaded according to their absolute orientation.

\section{Comparison to the elastic antiferromagnet on a triangular lattice}

Ising antiferromagnets (Ising models with a positive spinspin coupling constant $J$ ) are often used as models of the order-disorder transition in alloys. "Ordering" an alloy means that unlike particles prefer to be neighbors just like antiparallel spins in an antiferromagnet. But many metallic solid solutions form a fcc lattice, and antiferromagnetism does not "fit" the fcc lattice in the sense that the lattice cannot be filled up with alternating rows of up and down spins in all directions. This mismatch leads to "frustration"-i.e., a disordered lowest-energy state.

The two-dimensional analog of this type of system, the antiferromagnet on a triangular lattice, has been studied extensively $[8,15]$. In this model, spins are free to move around their lattice position and the neighboring spin-spin interaction parameter $J(i, j)$ is a function of the distance between the spins $i$ and $j$. In addition, there is a linear elastic term in the Hamiltonian with the strain depending on the spin positions, introducing the additional requirement of a slowly varying displacement field. It has been shown that coupling

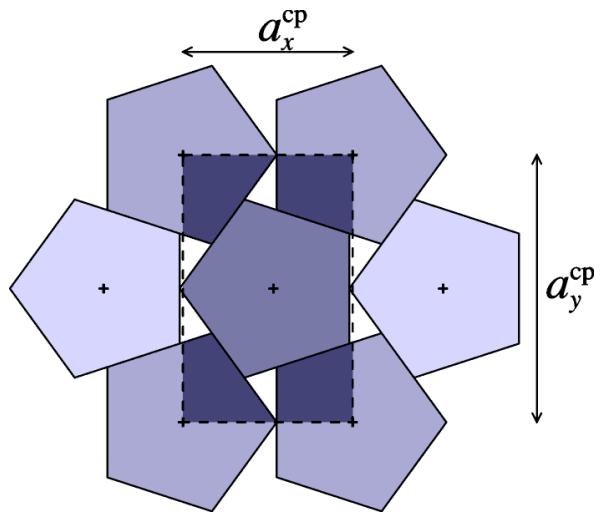

FIG. 5. Close packing: pentagons pack onto a distorted triangular lattice. The unit cell is shown as the rectangular area with darker shades; its dimensions and particle positions are given by Eqs. (7) and (8), respectively. to the strain of the lattice (by introducing compressibility) removes the frustration and allows for a transition to an ordered ground state [8]. This new phase, the "stripe" phase, has two broken symmetries: the Ising symmetry and the three-state symmetry of the underlying lattice.

The transition to this stripe phase has been found to be strongly first order under the assumption of a mean strain field [8]. When fluctuations in the spin positions are taken into account, the transition is still strongly first order for low values of the spin-displacement coupling constant relative to the elastic constants, but becomes weakly first order for higher values of the spin-displacement coupling constant [16].

The high-density phase transition in the pentagon system resembles this transition. Frustration due to the mismatch in particle and lattice geometry is removed by straining the lattice into the nonhexagonal, striped phase shown in Figs. 4 and 5. Here, the symmetries that are broken are the left-right symmetry (the direction where a pentagon corner is pointing in a bond direction) and a threefold symmetry involving the choice of direction of the stripes. The size of the density gap and the behavior of the order parameters $\alpha$ and $\Phi_{10}^{*} \Psi_{6}$ suggest a moderately weak first-order transition similar to what

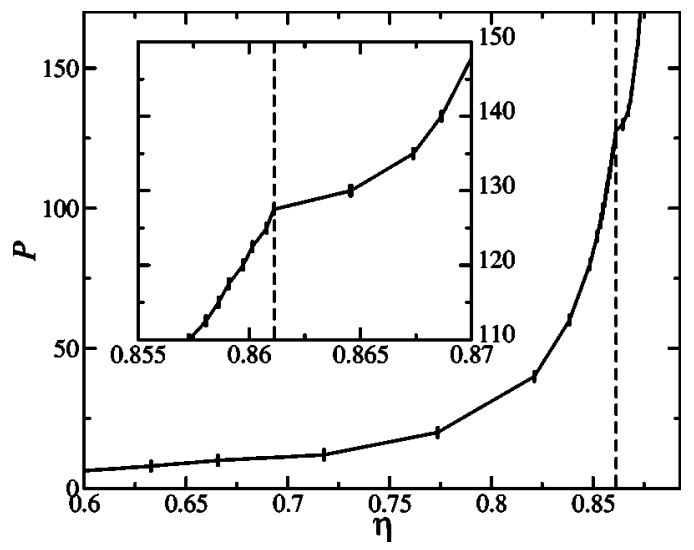

FIG. 6. Pressure $P$ versus packing fraction $\eta$ for heptagons: The equation of state shows a discontinuity at a larger packing fraction than for the pentagon case. 


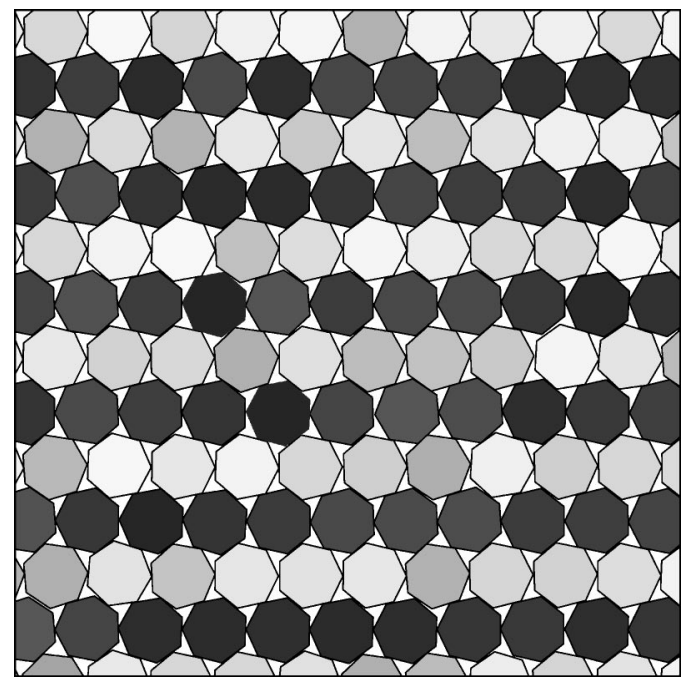

FIG. 7. Snapshot of the high-density phase of hard heptagons $(P=180)$. The particles are color coded according their absolute orientations.

is seen by Gu et al. [16] for the intermediate regime of the spin-displacement coupling constant strength.

\section{Changing the couplings: Heptagons}

Gu et al. have predicted that the transition to the striped phase becomes sharper with increasing effective coupling $\mu=\epsilon^{2} / E$, where $\epsilon$ is the coupling between the spins and displacements and $E$ is the elastic constant of the triangular lattice. In hard-particle systems one is not able to independently vary the direct orientational coupling between the particles and coupling to the lattice. The only parameter we can vary is the shape of the particles. In order to assess the effect of such a change on the phase behavior we have therefore also simulated hard heptagons.

Figure 6 shows the equation of state of hard heptagons. The high-density transition has shifted to a higher packing fraction (from $\eta=0.84$ for pentagons to $\eta=0.87$ ). A snapshot, Fig. 7, of the high-density phase reveals that is again a striped phase, with a lattice structure similar to that of the pentagons.

The equation of state (see inset of Fig. 6) and the order parameters (see Fig. 8), however, both clearly show that the transition has become weaker. Comparing to the parameter $\mu$ we see that this is naively consistent with the fact that (i) the effective orientational coupling between the heptagons is less than that of the less circular pentagons and (ii) the Young modulus of the lattice is larger due to the higher packing fraction achieved. However, a more detailed theory that maps the two models onto each other would be necessary to resolve the nontrivial density dependence of these effective interaction parameters.

\section{CONCLUSIONS}

We have investigated the phase behavior of hard pentagons and heptagons within Monte Carlo simulations. Penta-

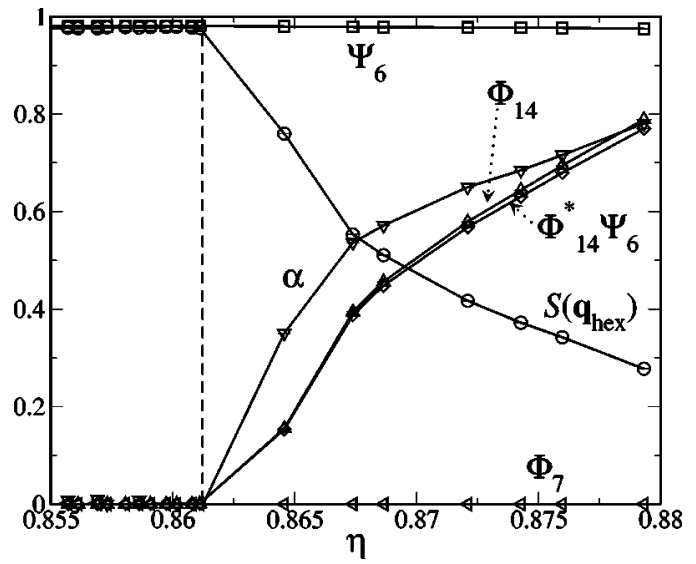

FIG. 8. Order parameters at the high-density transition in heptagons.

gons are a simple model of particles, whose geometry interferes with the symmetries allowed for crystals.

At low pressures pentagons exhibit a positionally and orientationally disordered isotropic phase. On increase of pressure they undergo a first-order phase transition to a positionally ordered, but orientationally disordered rotator phase. This transition proceeds like the freezing transition in hard disks. We do not find traces of fivefold symmetry in the orientational order parameters, contrary to the observations of Ref. [4] for cyclic pentamers, where it is reported that the molecular rotation is hindered in the hexagonal phase.

On further increase of pressure the nonspherical shape of the particles begins to influence the phase behavior. They undergo a first-order phase transition, in which the orientational degrees of freedom are to a large extent "frozen in" and the lattice becomes distorted. Both effects thus have their onset at the same transition.

As pentagons cannot be packed densely onto a regular triangular lattice, but can onto a distorted one, the positional order changes at this phase transition. The resulting distortion of the lattice alleviates frustration of the orientational and positional order. This effect is well known in the context of the antiferromagnetic Ising model. The transition to the high-density phase in hard pentagons resembles the transition to the striped phase in the antiferromagnetic case.

A short study of hard heptagons reveals that the mismatch between particle shape and crystal structure symmetry is, as one expects, less severe than in the pentagon case, resulting in a shift of the transition to the striped phase to higher densities and a weakening of its first-order character.

\section{ACKNOWLEDGMENTS}

This work is part of the research program of "Stichting Fundamenteel Onderzoek der Materie" (FOM) which is financially supported by "Nederlandse Organisatie voor Wetenschapelijke Onderzoek" (NWO). T.S. acknowledges support through a European Community Marie Curie grant (No. HPMF-CT-2001-01212). 
[1] M. P. Allen, G. T. Evans, D. Frenkel, and B. M. Mulder, in edited by I. Prigogine and S. A. Rice, Advances in Chemical Physics, (Wiley Interscience, New York, 1993), Vol 86.

[2] J. P. Hansen and I. R. McDonald, Theory of Simple Liquids (Academic Press, London, 1997).

[3] A. C. Brańka and K. W. Wojciechowski, Phys. Lett. 101, 349 (1984).

[4] A. C. Brańka and K. W. Wojciechowski, Mol. Phys. 72, 941 (1991).

[5] A. C. Brańka and K. W. Wojciechowski, Mol. Phys. 78, 1513 (1993)

[6] A. C. Brańka and K. W. Wojciechowski, Phys. Rev. Lett. 50, 846 (1983).

[7] K. W. Wojciechowski, K. V. Tretiakov, and M. Kowalik, Phys. Rev. E 67, 036121 (2003).
[8] Z.-Y. Chen and M. Kardar, J. Phys. C 19, 6825 (1986).

[9] M. Parrinello and A. Rahman, Phys. Rev. Lett. 45, 1196 (1980).

[10] K. W. Wojciechowski and K. V. Tretiakov, Comput. Phys. Commun. 121, 528 (1999).

[11] K. V. Tretiakov and K. W. Wojciechowski, J. Phys.: Condens. Matter 14, 1261 (2002).

[12] Katherine J. Strandburg, Rev. Mod. Phys. 60, 161 (1988).

[13] C. L. Henley, Phys. Rev. B 34, 797 (1986).

[14] Y. Limon Duparcmeur, A. Gervois, and J. P. Troadec, J. Phys.: Condens. Matter 7, 3421 (1995).

[15] G. H. Wannier, Phys. Rev. 79, 357 (1950).

[16] L. Gu, B. Chakraborty, P. L. Garrido, M. Phani, and J. L. Lebowitz, Phys. Rev. B 53, 11985 (1996). 